\title{
Juristinnen in der DDR
}

Anlässlich der Herausgabe des „Juristinnenlexikons“ im Jahre 2005 und der damaligen Recherchen stellten wir fest, dass über DDR-Juristinnen - im Gegensatz zu DDR-Juristen - wenig bekannt ist. Einzige Ausnahme: Hilde Benjamin, die offenbar potentiellen Jurastudentinnen regelmäßig zur Abschreckung vorgeführt wurde. Zitat: „Willst du werden wie Hilde Benjamin?“ Das soll sich mit der Ausstellung ändern.

Das Projekt will darüber hinaus - insbesondere in einer wissenschaftlichen Begleitpublikation - die Frage beantworten, ob die ostdeutsche Justiz tatsächlich „Schauplatz einer weiblichen Erfolgsgeschichte“ war, weil die DDR einen weltweit einmalig hohen Anteil von Frauen unter den Juristen hatte. Denn dieser Anteil hing auch in der DDR in den verschiedenen juristischen Berufsgruppen stark mit deren Prestige zusammen. In der sozial höher angesiedelten Anwaltschaft waren Frauen nur in geringer Zahl vertreten. Von den Notaren jedoch, die in der DDR einen eher niedrigen sozialen Status innehatten, waren zum Zeitpunkt der deutschen Einheit 67,8 Prozent weiblich. Auch der Anteil von Frauen in der Justiz wurde kleiner, je höher die Position in der Justizhierarchie war. 1979 stellten sie zwar 51,1 Prozent der Richter an den Kreisgerichten, aber nur 23,2 Prozent der Direktoren. Auch in der DDR griffen damit offenbar alte Mecha- nismen, die zu einem deutlichen Ungleichgewicht zwischen den Geschlechtern führten.

Eine Wanderausstellung soll anhand von Tafeln mit 12 Einzelbiografien von Juristinnen

- aus verschiedenen „Generationen“: 1. vor Ende des 2. Weltkriegs ausgebildete Juristinnen, 2. in der DDR ausgebildete Juristinnen, die auch ausschließlich in der DDR berufstätig waren (dazu gehören insbes. die Volksrichterinnen), 3. in der DDR ausgebildete Juristinnen mit geteilter Berufsbiografie und

- Berufsgruppen: Richterin, Rechtsanwältin, Notarin, Staatsanwältin, Wissenschaftlerin, Verwaltungsjuristin (auch Polizei, Armee, Repressionsapparat), Justiziarin

einen Einblick in die Ausgestaltung der juristischen Berufe in der DDR aus der Genderperspektive ermöglichen. Die Biografien werden durch allgemeine Tafeln mit Hintergrundinformationen zur Situation der DDR-Justiz und der Frauen in der DDRJustiz sowie im Berufsleben ergänzt.

Die rechtshistorische Forschung soll in einer begleitenden Publikation vertieft und auch anhand von Statistiken, Fallstudien usw. sehr viel detaillierter werden, als dies auf den Ausstellungstafeln möglich ist.
Wenn Sie diese Möglichkeit nutzen möchten, so gelangen Sie unter www.djb.de/Spenden dorthin.

\section{Sponsoring durch Kanzleien oder Unternehmen}

Der Bundesvorstand hat im Jahre 2008 beschlossen, auch moderne Arten der Finanzierung wie das Sponsoring zu nutzen. Beim Sponsoring werden Finanzmittel, Sachleistungen und Dienstleistungen seitens des Sponsoring-Gebers (in der Regel ein Wirtschaftsunternehmen) gegen Image- und Know-howTransfer, Kommunikationsleistungen, Logoplatzierungen, Emotionalisierungen sowie verkaufsfördernde Maßnahmen seitens des Sponsoring-Nehmers ausgetauscht. Dieser Austausch basiert regelmäßig auf einem klaren Vertrag.
Sollten Sie in Ihrer Kanzlei oder in Ihrem Unternehmen für die Vergabe von Sponsoring-Geldern verantwortlich sein, würden wir uns freuen, wenn Sie mit uns Kontakt aufnehmen. Sponsoringbeauftragte ist Vizepräsidentin Renate Maltry (E-Mail: renate.maltry@djb.de).(Renate Maltry, Dagmar Brinkmann)

Für den djb danke ich den bisherigen Spenderinnen ganz herzlich! Insbesondere der Realisierung unseres Gedenktafel-Projekts sind wir einen großen Schritt näher gekommen.

Jutta Wagner

Präsidentin

\section{Ausschreibung}

\section{Arbeitsstab „Berufsorientierung, Karriereplanung, Mentoring“ \\ - Vorsitz und Mitgliedschaft -}

Der Arbeitsstab „Berufsorientierung, Karriereplanung, Mentoring “ sucht zur Betreuung vorhandener und der Entwicklung neuer Projekte eine Vorsitzende und weitere Mitglieder.

Informationen über die bisherige Arbeit sind auf der djbWebseite abrufbar: http://www.djb.de/karriere/arbeitsstab-berufsorientierung-karriereplanung-mentoring/
Kolleginnen, die mitarbeiten möchten, werden gebeten, sich für den Vorsitz und/oder die Mitgliedschaft schriftlich bis zum 15. April 2009 über die Geschäftsstelle beim Bundesvorstand zu bewerben. Die Bewerbung sollte in kurzer Form Angaben darüber enthalten, welche Projekte Sie interessieren und vielleicht auch selbst vorschlagen möchten. 